\title{
Klaus Bohndorf, Mark Anderson, Mark Davies, Herwig Imhof, Klaus Woertler: Imaging of bones and joints. A concise, multimodality approach
}

\author{
Georg Thieme Verlag KG 2016. ISBN 978-3-13-240647-6
}

\author{
Andrea Genova $^{1} \cdot$ Luigi Mansi ${ }^{2}$
}

Published online: 30 October 2017

(C) Springer-Verlag GmbH Germany 2017

This book is an adapted and revised version of the third German edition that concentrates on the principles of musculoskeletal imaging. The editors are Klaus Bohndorf, Professor of Radiology at the Medical University of Vienna (Austria), Mark W. Anderson, Professor of Radiology at the University of Virginia in Charlottesville (US), Mark Davies, Professor of Radiology at the Royal Orthopedic Hospital in Birmingham (UK), Herwig Imhof, formerly Professor of Radiology at the Medical University of Vienna (Austria), and Klaus Woertler, Professor of Radiology at the Technical University of Munich (Germany), who utilized the contributions of 35 specialists from Germany, Austria, United Kingdom and US.

The book consists of 12 chapters, covering the imaging of the various disorders of bones and joints. The first two chapters are devoted to acute trauma and overuse injuries, starting with the essentials and going deep according to the region. The other chapters focus on infection of bones and joints, tumors, bone marrow, osteonecrosis, osteochondrosis, metabolic and hormonal bone disorders, congenital disorders of the skeletal development, rheumatic disorders, and miscellaneous bone and joint disorders. The last chapter provides a

Luigi Mansi

mansi.luigi@libero.it

Università Federico II, Naples, Italy

2 Centro Interuniversitario per lo Sviluppo Sostenibile (CIRPS), Roma-Napoli, Italy discussion about the interventions involving bones and joints, with indications, contraindications, technique and complications of some of the most relevant surgical operations regarding bones and joints.

Each chapter has the same format: starting from anatomy, you will find the most important features of the region of interest, followed by pathology and pathophysiology of each disease, described with its clinical presentation. Then the patient is analyzed through radiological (Rx, US, CT, MRI) and, when appropriate, nuclear medicine findings. Special features of child disorders and the most important differential diagnoses are also discussed. Particularly noteworthy is the special layout of this book. The material is presented in units of two facing pages, with the left page for text and the right page for images, including the description of the imaging plane and, in the case of MRI, of the imaging sequence. This solution allows the reader to have an immediate feedback to maximize his/her comprehension. Plus this book focuses on more than 2100 images (!) in about 400 pages, further implemented by an excellent section of e-contents: 338 images, accompanied by a supplemental text and references, that are available online on Thieme Media Center. The clinical interest for each of these further images is clearly indicated in the book by the use of an icon.

Definitely this book can be very useful for all the specialists interested in bone and joints. It means that it can be warmly suggested not only to radiologists and nuclear physicians, but also to orthopedists and to all the others who, as professional or student, may be interested in the imaging of bones and joints. 